\title{
Article \\ ESWT Enhances Expression of Pdia-3 which is a Key Factor of 1a,25-Dihydroxyvitamin D3 Rapid Membrane Signaling Pathway in Treatment of Early Osteoarthritis Knee
}

\author{
Shan-Ling Hsu ${ }^{1,2, *}$, Jai-Hong Cheng ${ }^{1,3, \dagger}$, Ching-Jen Wang ${ }^{1,2}$, Jih-Yang Ko ${ }^{1,2}$ \\ and Chih-Hsiang Hsu ${ }^{2}$ \\ ${ }^{1}$ Center for Shockwave Medicine and Tissue Engineering, Kaohsiung Chang Gung Memorial \\ Hospital and Chang Gung University College of Medicine, Kaohsiung 833, Taiwan; \\ cjh1106@cgmh.org.tw (J.-H.C.); w281211@cgmh.org.tw (C.-J.W.); \\ kojy@cgmh.org.tw (J.-Y.K.) \\ 2 Department of Orthopedic Surgery, Kaohsiung Chang Gung Memorial Hospital and Chang Gung \\ University College of Medicine, Kaohsiung 833, Taiwan; hcs027@cgmh.org.tw \\ 3 Medical Research, Kaohsiung Chang Gung Memorial Hospital and Chang Gung University \\ College of Medicine, Kaohsiung 833, Taiwan \\ * Correspondence: hsishanlin@yahoo.com.tw \\ $\uparrow$ These authors have contributed equally to this work.
}

\begin{abstract}
Dysregulation of cartilage homeostasis and the changes in the density and the architecture of the subchondral bone were postulated as a potent mechanically pathological activity contributing to osteoarthritis (OA) pathogenesis. Extracorporeal shockwave therapy (ESWT) is a new, none invasive and effective method in the treatment of animal OA model. In the current study, we demonstrated that shockwave induced the expression of protein-disulfide isomerase-associated 3 (Pdia-3) which is a multifunctional protein hypothesized to be a significant mediator for $1 \alpha, 25$-Dihydroxyvitamin $\mathrm{D} 3\left(1 \alpha, 25(\mathrm{OH})_{2} \mathrm{D}_{3}\right)$ signaling pathway using two-dimensional electrophoresis. Histological analysis and quantitative polymerase chain reaction (qPCR) were verified and observed that the expression of Pdia-3 at 2 weeks was significantly higher than that of any other group at 4 weeks, 8 weeks, and 12 weeks post-shockwave treatment in early OA knee of rat. The other factors of the $1 \alpha, 25(\mathrm{OH})_{2} \mathrm{D}_{3}$ rapid membrane signaling pathway including extracellular signal-regulated protein kinases 1 (ERK1), osteopontin (OPG), alkaline phosphatase (ALP), and matrix metallopeptidase 13 (MMP13) were measured and significantly increased by qPCR at 2 weeks post-shockwave treatment in early OA knee. Our proteomic data revealed significant Pdia-3 expression in microenvironments of joint tissue that could be actively responded to ESWT, which may potentially regulate biological function of chondrocytes and osteoblasts in the treatment of OA knee.
\end{abstract}

Keywords: protein-disulfide isomerase-associated 3; osteoarthritis; extracorporeal shockwave therapy; 1 $\alpha, 25$-Dihydroxyvitamin D3 signaling pathway; two dimensional electrophoresis

\section{Introduction}

Osteoarthritis (OA), one of the most common causes of musculoskeletal disorders in the developed countries [1], is characterized by cartilage attrition, reduced subchondral bone remolding, osteophyte formation and synovial inflammation, and factors inducing cartilage 
degeneration such inappropriate mechanical load [2], disturbed biochemical regulation [2] and genetic mutation[3] are potential etiologic causes of OA.

Osteoarthritis had been considered to be primarily cartilage disorder characterized by cartilage degradation. Intensive inflammatory cytokines such as interleukin, tumor necrosis factor and proteases secreted from joint component cells caused by abnormal mechanical force are mainly attributable to accelerate cartilage damage, loss of compensatory synthesis and eventually deteriorate the function of extra-cellular matrix organization[4-6]. Dysregulation of cartilage homeostasis caused by intensive chondrocyte apoptosis has been reported as potent pathological activity in the development of osteoarthritis. Disturbance of oxidative stress [7], proapoptotic/antiapoptotic regulation[8, 9] and mitochondrial dysfunction [10] have been proposed to modulate chondrocyte survival in the progression of osteoarthritis. However the molecular mechanism by which chondrocyte propagates toward to programming cell death is not clearly defined.

1 $\alpha, 25$-Dihydroxyvitamin $\mathrm{D} 3\left(1 \alpha, 25(\mathrm{OH})_{2} \mathrm{D}_{3}\right)$ is essential in calcium homeostasis to regulation of endochondral ossification [11]. In vitamin D deficiency, bone matrix synthesis and cartilage growth are inhibited. It has been investigated action on osteoblasts and growth plate chondrocytes through classic unclear vitamin D receptor (VDR) and Pdia-3. Pdia-3 was demonstrated to a key factor in $1 \alpha, 25(\mathrm{OH})_{2} \mathrm{D}_{3}$ induced phospholipase A2 (PLA2) and protein kinase $\mathrm{C}(\mathrm{PKC})$ activation and downstream responses of gene transcription [12, 13].

A growing body of evidence has demonstrated ESWT promoted tissue repair in various tissue and initiated biological responses [14, 15]. Reportedly ESWT ameliorated experimental osteoarthritic cartilage damage and alter angiogenesis pattern [15]. The preliminary proteomic data revealed abundant significant proteins that warrant further characterization [16-18]. These proteins of interest were reported to participate in the cellular response to physical stress, calcium homeostasis, chemotaxis and lipid oxidative stress in several tissue types under pathological contexts. Therefore, we hypothesize that multiple molecules in joint tissue microenvironments may be actively responded to ESWT treatment, which potentially regulates survival and biological function of chondrocytes in OA knee. To test the null hypotheses, we conducted studies to delineating the active responsive molecules in ESWT-regulated biological responses using comparative proteomic technique helps the construct of molecular mechanism of alleviation of OA and whether ESWT changed signaling interfering joint microstructures. Based on these translational experimental data, we could further explore a new regime with good potential for rescuing OA knee joint injury.

\section{Results}

\subsection{The effect of shockwave therapy on articular cartilage}

In the macroscopically normal articular cartilage, some changes of cell distribution in the superficial layer were noticed. Furthermore, the differences between OA knee and shockwave treatment were pronounced. After application of ESWT to the subchondral bone of the medial tibia condyle showed regression of osteoarthritis of the knees in rats (Figure 
1A). In the OA+ESWT group of knee, the articular cartilage, which Mankin's score ranged from 2 to 5, was better preserved than in the OA group, which Mankin's score ranged from 4 to 8 during 2 weeks to 12 weeks (Figure 1B). In the OA group, significantly greater articular cartilage degeneration was evident and evaluated in wide Mankin's score range.

2.2. Two-dimensional electrophoresis in shockwave knee compared with osteoarthritic knee at 2 weeks after index surgery

Proteome analysis of the left knee was conducted by two-dimensional gel electrophoresis and mass spectrometry. Protein spots in gel were developed by silver stains and scanned by an Amersham ImageScanner. The level of significant spot was 1.5 fold increase or decrease. Twelve spots were found to be differentially abundant, including 9 spots decreased intensity and 3 spots increased signal observed in OA+ESWT group when compared with normal control (NC) group and OA group at 2 weeks after index surgery. (Figure 2 and Supplemental Figure 1)

The 8 identified proteins were significantly different between the shockwave with OA knee and OA knee as well as also changed with disease development (Figure 3). Among those, 2 proteins were up-regulated including Pdia-3 and guanine nucleotide-binding protein subunit beta-2-like. Moreover, six proteins were identified and suppressed in OA+ESWT group, including Beta-enolase, chloride intracellular channel protein 1, malate dehydrogenase, purine nucleoside phosphorylase, creatine kinase M-type and L-lactate dehydrogenase A chain (Table 1). These proteins were involved in regulating various cellular functions, including cytoskeletal structure, ion channel components, energy metabolism, and protein degradation. These findings indicated altered protein expression in the pathogenesis of OA and illuminated a novel therapeutic avenue for treatment in OA disease. Among these proteins, protein Pdia-3 in the knee joint, which was reported to osteoblast and chondrocyte cells integrity and function [19-21].

\subsection{The expression of Pdia-3 and extracellular signal-regulated kinases 1 (ERK1) after ESWT on early OA knee}

The spot of Pdia-3 observed difference between OA+ESWT group and OA group at 2, 4 , 8 and 12 weeks after index surgery were first destaining and enzymatic in gel digestion and then subjected to MALDI/TOF mass spectrometry analysis. The expression of Pdia-3 at each time course was summarized in Figure 4. Significantly, regulation of the mRNA of Pdia-3 at each time course was noted in OA+ESWT group compared with OA group and NC group. It was especially enhanced to 13 fold at 2 weeks than at 4,8 and 12 weeks after shockwave treatment with OA knee (Figure 4A).

Extracellular signal-regulated kinases (ERKs), acted as an integration point for multiple biochemical signals, and were involved in an osteoblast cellular processes such as proliferation, differentiation, transcription regulation and development $[12,22]$. Upon activation by Pdia-3 after ESWT, these kinases translocations to the nucleus of the osteoblast cells, where it phosphorylated nuclear targets. Two alternatively spliced 
transcript variants encoding different protein isoforms had been described for this gene. Significant increases of ERK1 in OA+ESWT group as compared with OA and NC groups were found, especially at 2 weeks (Figure 4B).

\subsection{The expression of related gene of rapid membrane signaling pathway after ESWT}

The addition of shockwave therapy to knee resulted in increase of bone formation markers, including OPG, ALP and MMP13. The ALP increased if there was active bone formation occurring, as ALP was a byproduct of osteoblast activity [23, 24]. OPG in bone was the major determinant of bone mass and strength [25-27]. MMP 13 was established marker gene for bone formation [28, 29]. Significantly less pronounced subchondral bone remodeling with decreases in osteogenesis was noted in OA group compared with the OA+ESWT group. It appeared that application of ESWT to the medial tibia condyle of the knee improved osteogenesis and bone turnover rate of the subchondral bone in OA knees, and the results were comparable to that of the normal control in rats (Figure 4C, 4D and 4E).

\subsection{The effect of ESWT on Pdia-3 expression and extracellular matrix in articular cartilage and subchondral bone of $O A$ knee}

We further investigated expression of Pdia-3 from our microscopically IHC stain in articular cartilage and subchondral bone of knee at 2 weeks (Figure 5). We observed the expression of Pdia-3 was more $40 \%$ and $15 \%$ concentrated in OA+ESWT group than OA group and $\mathrm{NC}$ group (Figure 5A and 5B). The staining signal was particularly enriched on the superficial layer of cartilage and bone marrow of subchondral bone, indicated that Pdia-3 co-responded to ESWT in the chondrocyte and subchondral bone because the up-expression of Pdia-3 significantly increased on the chondrocyte and bone after OA+ESWT compared with $\mathrm{OA}$ and $\mathrm{NC}$ groups.

The synthesis of extracellular matrix of aggrecan and collagen type II was being investigated for their role in cartilage formation [5, 30, 31]. The OA+ESWT group showed significantly increased amount of the collagen type II (about 3 fold higher) and aggrecan (about 20 fold higher) at 2 and 4 weeks when compared with the $\mathrm{NC}$ and $\mathrm{OA}$ group $(\mathrm{P}<0.05)$. (Supplemental Figure 1A and 1B; Supplemental Table 1) 


\section{Figures, Tables and Schemes}

A.

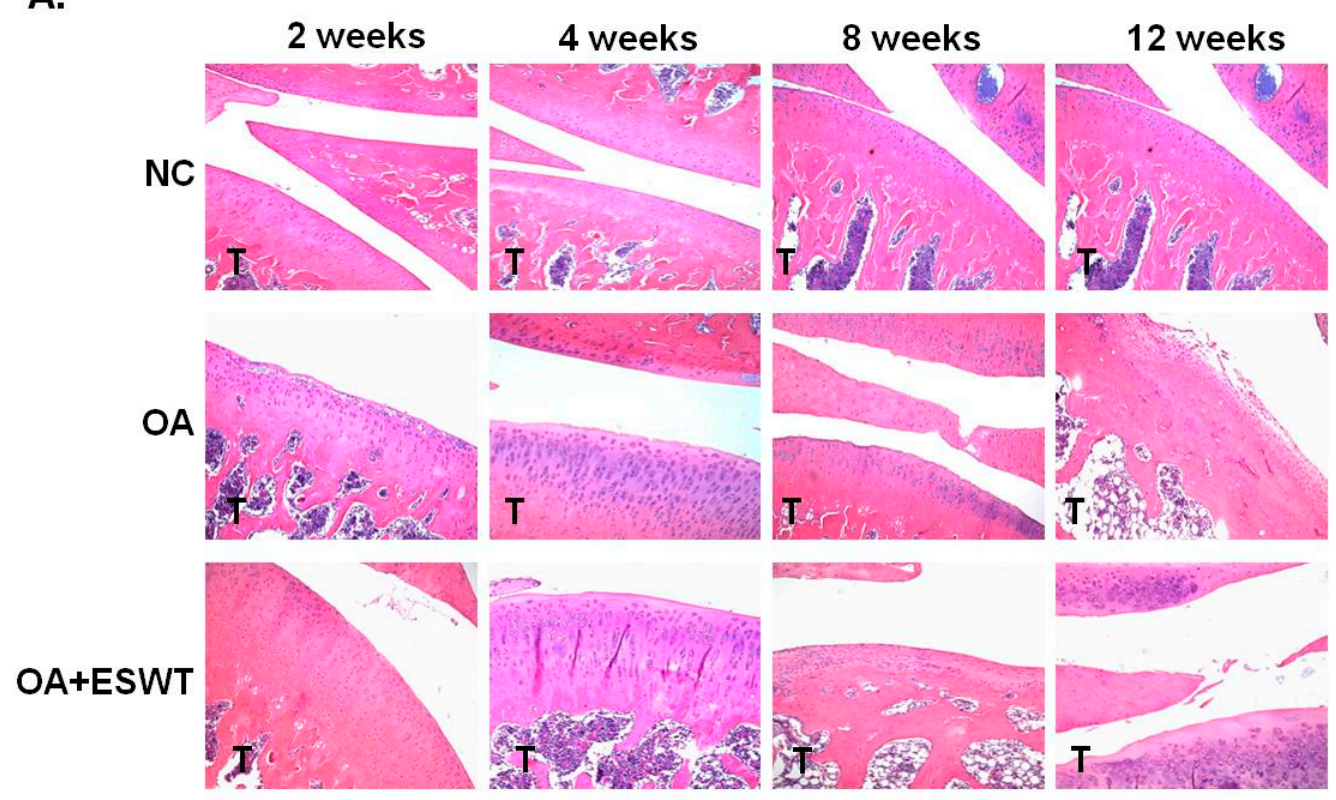

B.

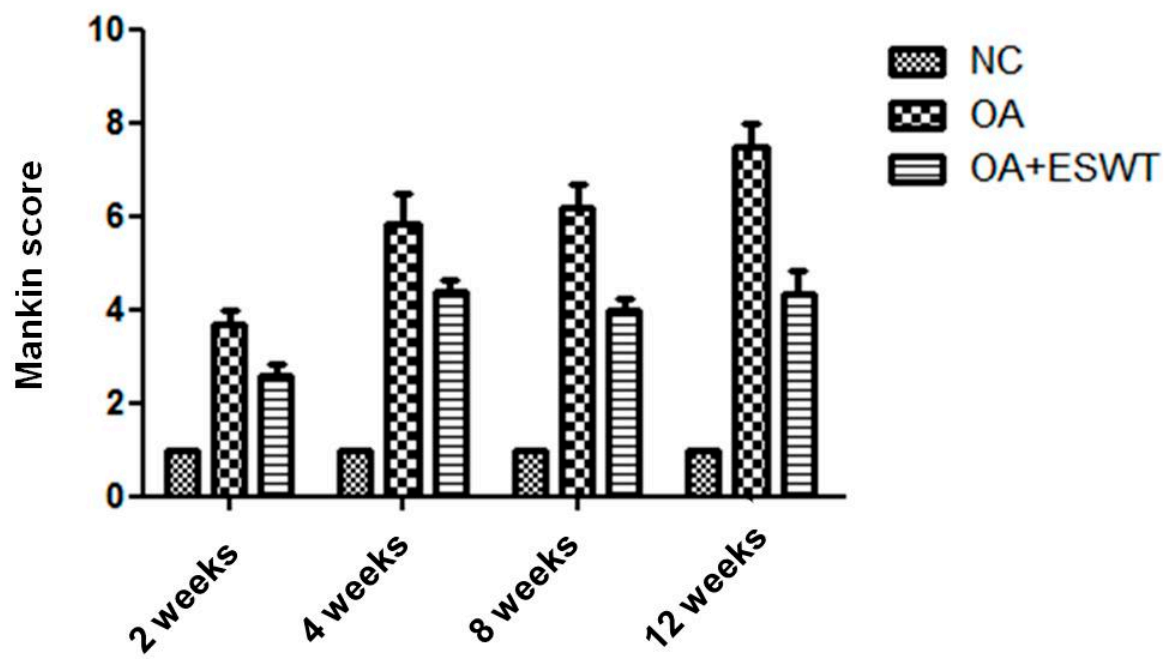

Figure 1. Histologic results of the early OA knee after ESWT. (A) Representative histological photographs of early OA knee after ESWT at 2, 4, 8, 12 weeks. Normal control (NC) showed normal structural integrity, cellularity, and cartilage height and tidemark integrity. (B) Scores across a section of normal cartilage ranged from 0 to 1 . OA knee of section with clefts and modest proteoglycan depletion was assigned scores ranging from 4 to 8 . The OA+ESWT group showing regression of $\mathrm{OA}$ change was given scores from 2 to 5 . $\mathrm{T}$ was indicated tibia. Specimens were stained by conventional hematoxylin-eosin. Specimens were observed in hundred-fold magnification. 
OA

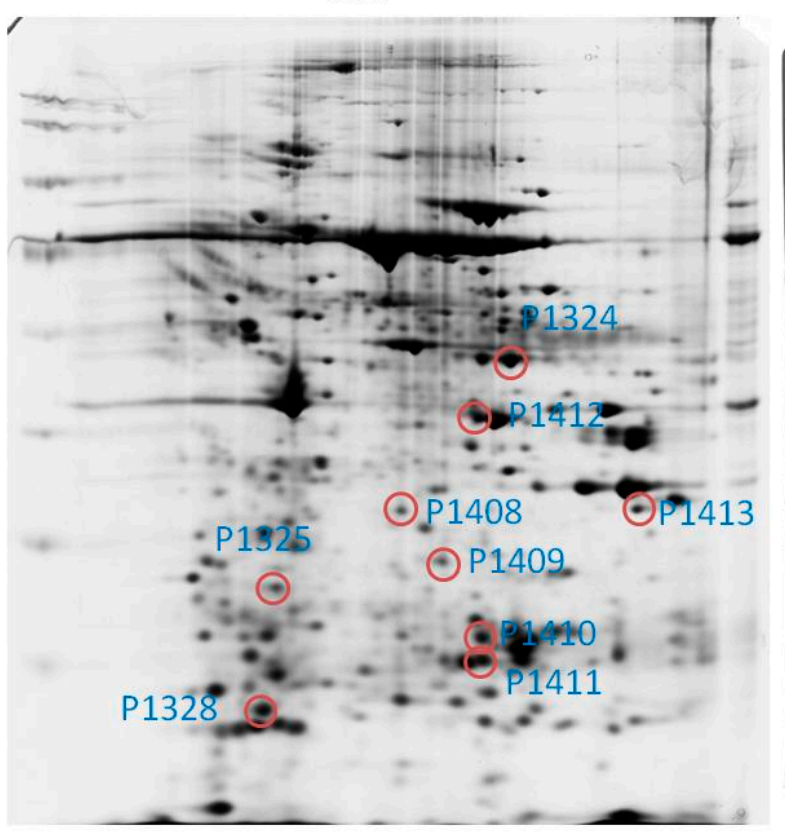

OA+ESWT

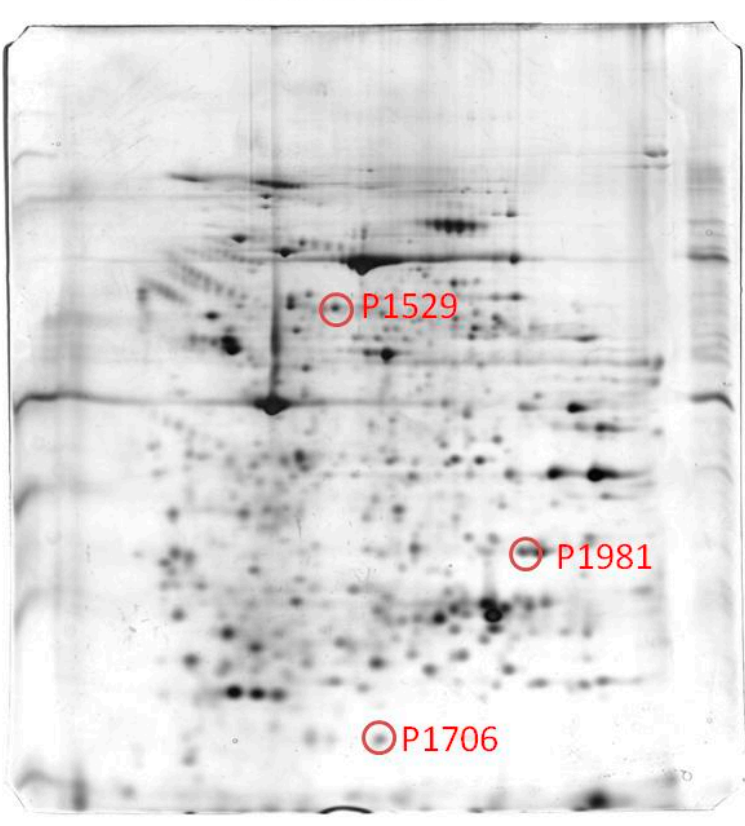

Figure 2. Protein spots of interest in two-dimensional gel electrophoretograms. ESWT induced or suppressed expression of several different proteins at 2 weeks. The level of significant spot was 1.5 fold increase or decrease. Twelve spots were found to be differentially abundant, including 9 spots decreased intensity and 3 spots increased signal observed in OA+ESWT group when compared with

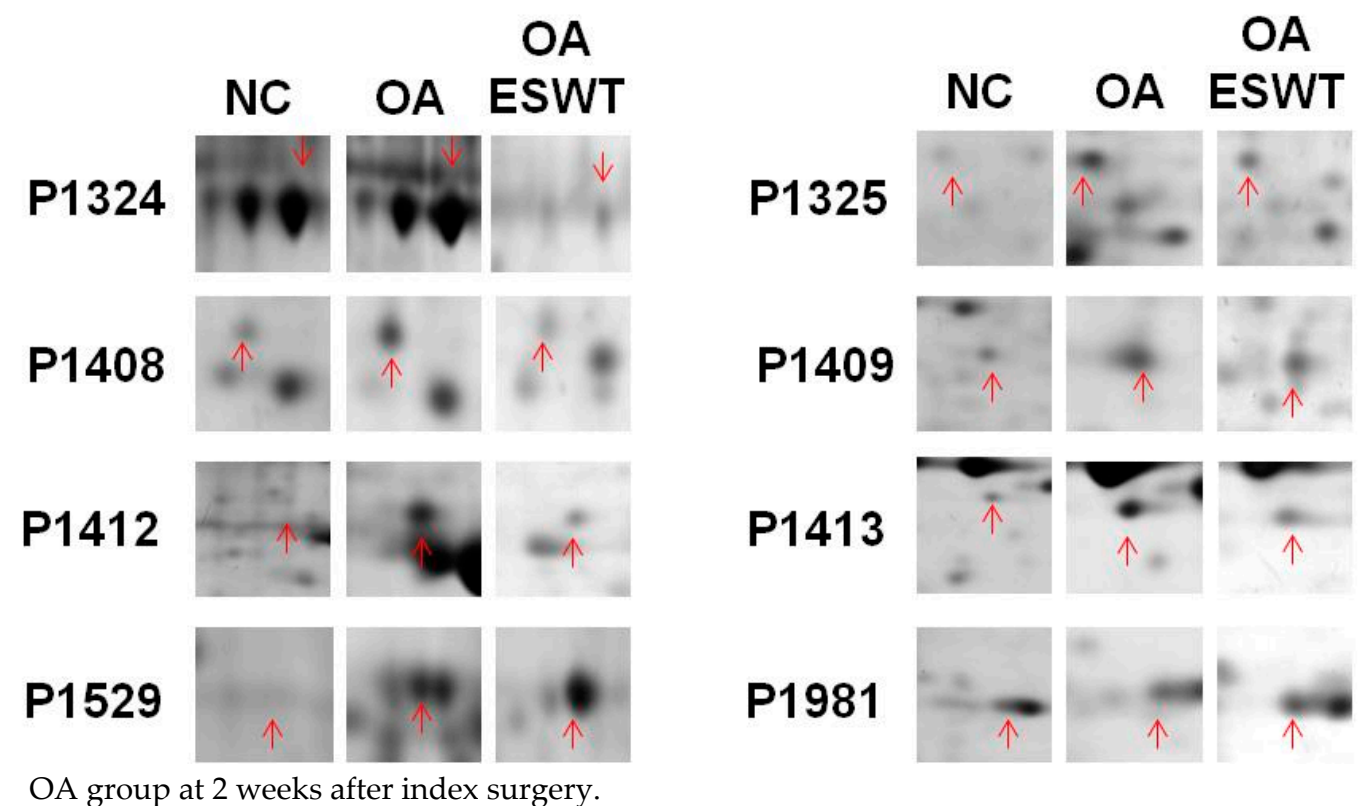

Figure 3. Enlarged the regions of the 8 spots of interest in the sliver-stained SDS-polyacrylamide gels. The arrows indicated the spot of interest between OA+ESWT and OA groups. ESWT promoted two proteins up-regulation including Pdia-3 (P1529) and guanine nucleotide-binding protein subunit beta-2-like (P1981). Moreover, six proteins were identified that shockwave knee suppressed, including Beta-enolase (P1324), chloride intracellular channel protein 1 (P1325), malate dehydrogenase (P1408), purine nucleoside phosphorylase (P1409), creatine kinase M-type (P1412) and L-lactate dehydrogenase A chain (P1413). Red arrow indicated the position of interest spots. 
A.

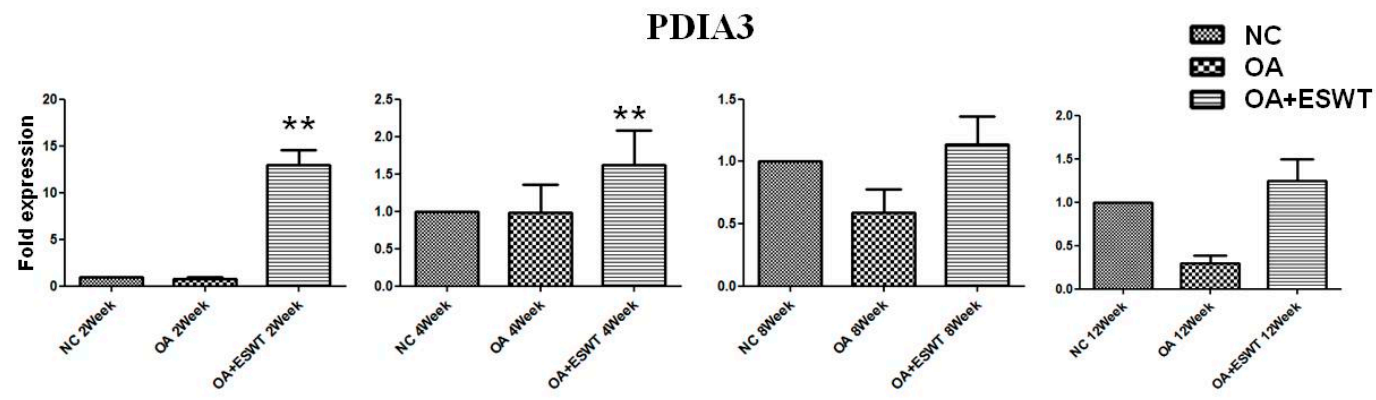

B.

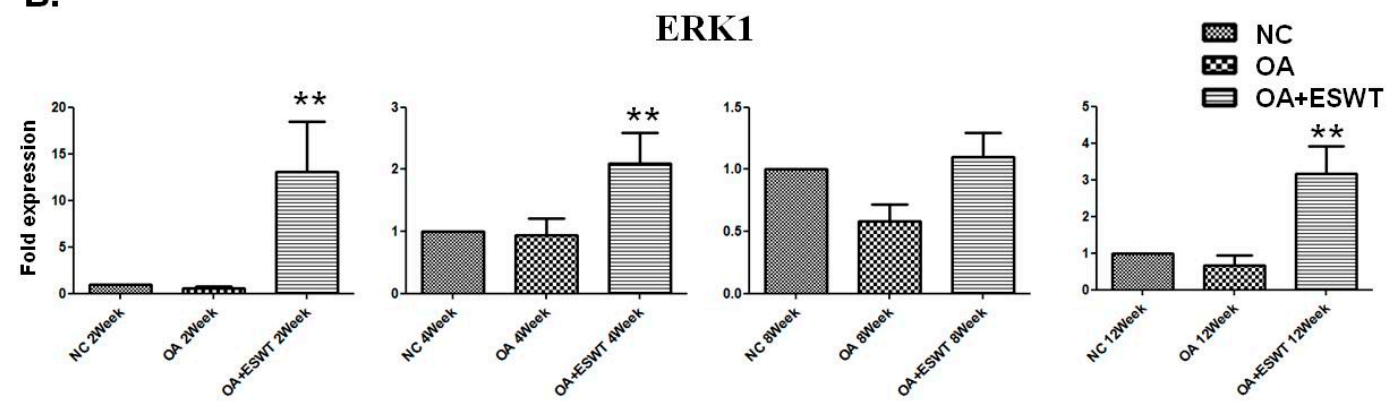

c.

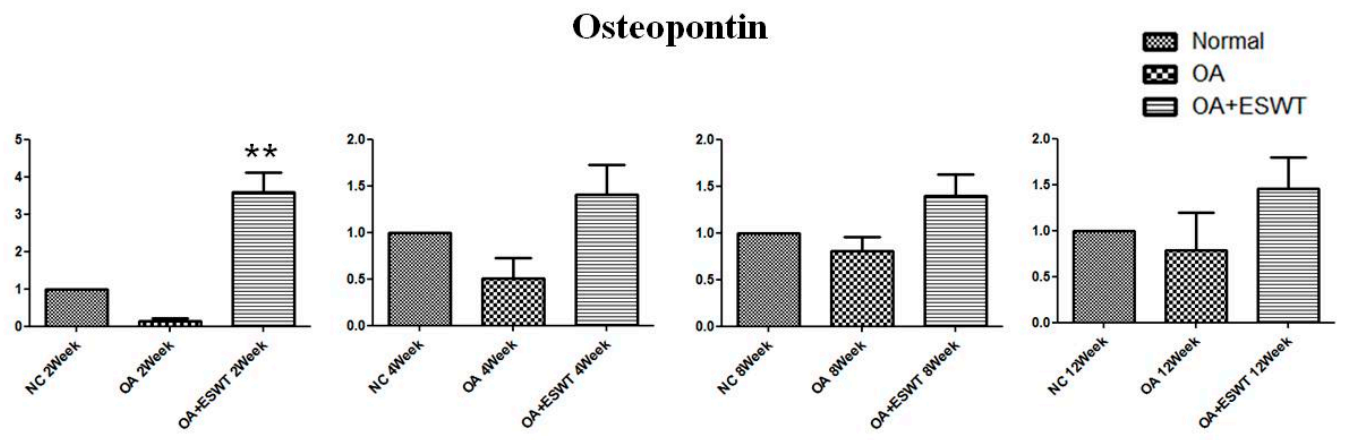

D.

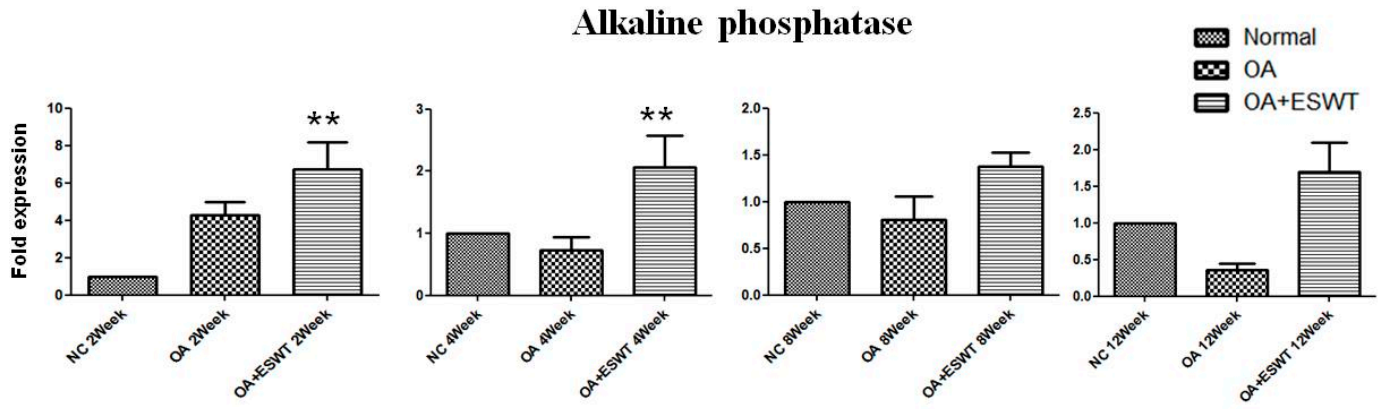

E.

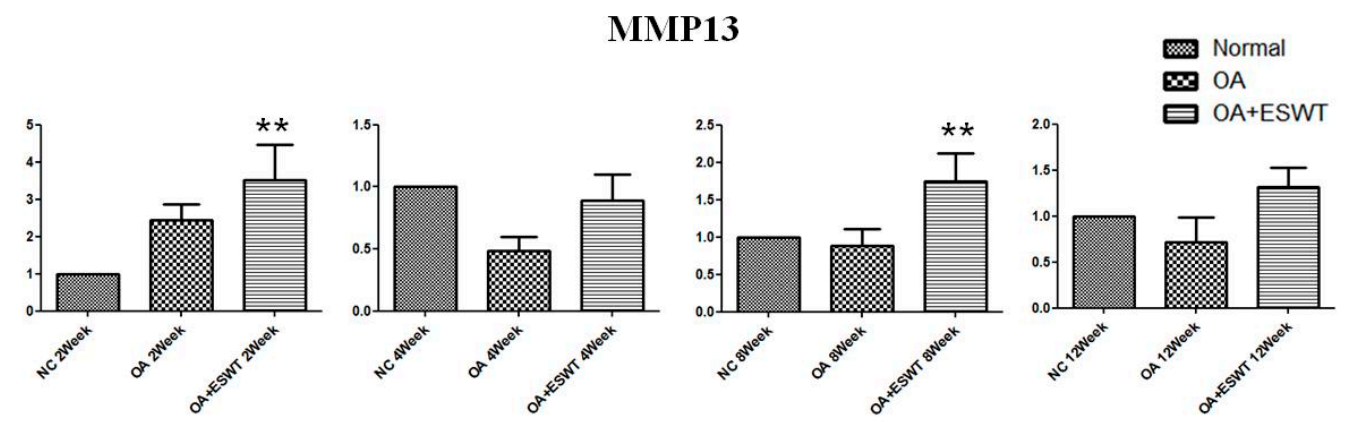


Figure 4. (A) Effect of ESWT on Pdia-3 expression in early OA knee. ESWT rapidly increased Pdia-3 activity at 2 weeks and then decreased at 4,8 and 12 weeks. When compared with OA knees after index surgery, it had significant difference at 2 and 4 weeks $(\mathrm{P}<0.05)$. (B) ESWT promoted ERK1 expression at 2 and 4 weeks as compared with OA knee after index surgery ( $P<0.05)$. (C-E) Effect of ESWT on bone formation markers in early OA knee. Shockwave therapy increased bone formation as implicated by activing OPG, ALP and MMP 13 intensity, especially at 2 weeks after treatment. $(\mathrm{P}<0.05)$ Real-time PCR was performed against 3 bone related genes: (C) osteoprotegerin; (D) alkaline phosphatase and (E) matrix metallopeptidase 13. The OA+ESWT group showed significant more amount of bone turnover rate than as compared with OA group. $(\mathrm{P}<0.05)$

A.

B.

Subchondral bone
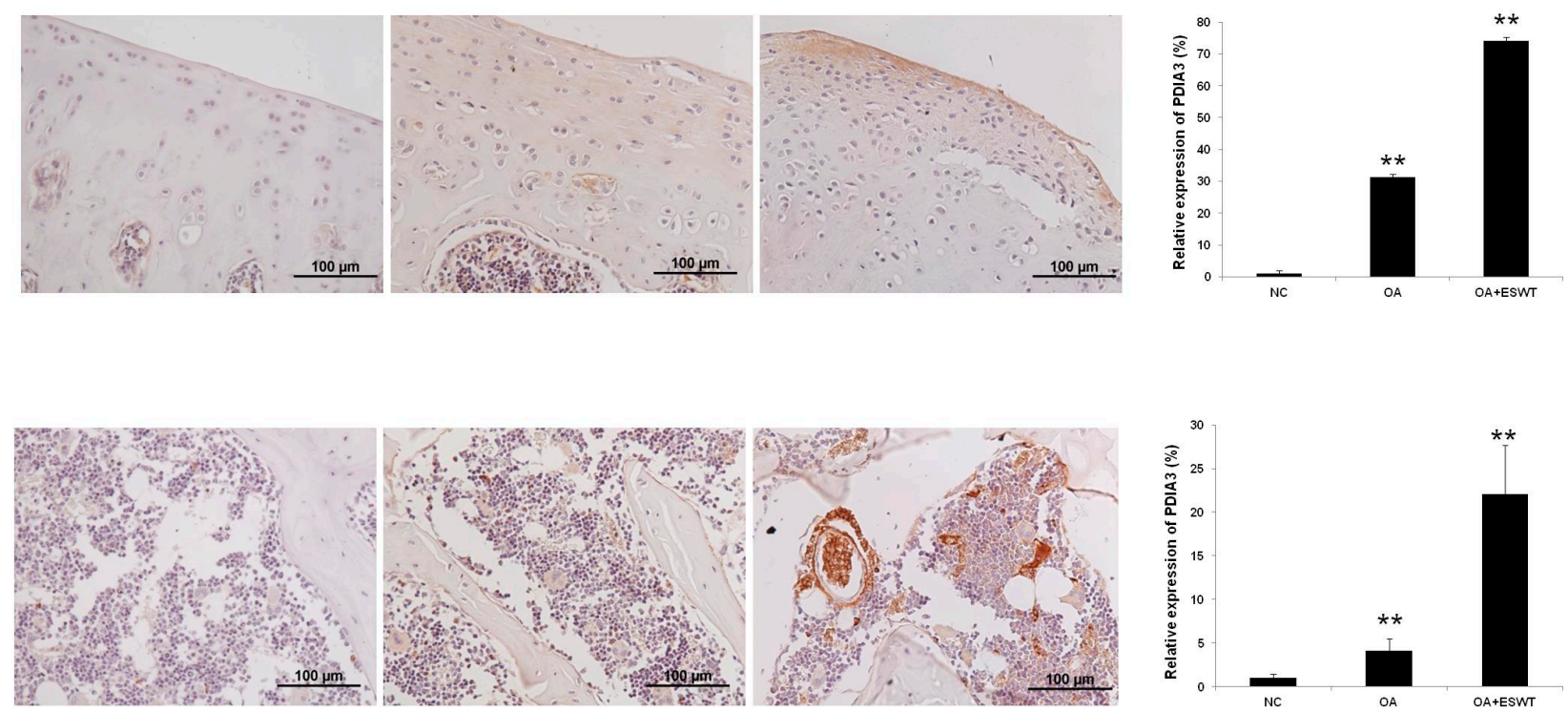

Figure 5. IHC staining of Pdia-3 in chondrocyte and subchondral bone with and without ESWT in early OA knee at 2 weeks. (A) The Pdia3 (brown color) was distributed over the cartilage and was enriched in the articular surface of OA+ESWT group. After quantization of spots by scanning densitometry, the OA+ESWT group showed significantly more chondrocyte expression of Pdia-3 compared with OA knee.( $\mathrm{P}<0.05)$. (B) NC group showed no staining in cell without primary Pdia-3 bodies. Cells were stained for antibodies against of Pdia-3 (brown spots) and imaged at 100 magnifications. After quantization of spots by scanning densitometry, the OA+ESWT group showed significantly more subchondral bone expression of Pdia-3 compared with OA group $(\mathrm{P}<0.05)$. 
Table 1. Characteristics of positively identified spots by mass spectrometry.

\begin{tabular}{|c|c|c|c|c|c|}
\hline Number & $\begin{array}{l}\text { Name of identified } \\
\text { protein }\end{array}$ & Entry name & Score & $\begin{array}{l}\text { Molecular } \\
\text { weight [kDa] }\end{array}$ & $\begin{array}{l}\text { Theoretical } \\
\text { PI value }\end{array}$ \\
\hline P1324 & Beta-enolase & ENOB_RAT & 603.1976361 & 47.33743 & 6.886547057 \\
\hline P1325 & $\begin{array}{l}\text { Chloride intracellular } \\
\text { channel protein } 1\end{array}$ & CLIC1_RAT & 260.7394264 & 27.30589 & 4.944812479 \\
\hline P1408 & Malate dehydrogenase & MDHC_RAT & 344.71 & 36.46005 & 6.168189325 \\
\hline P1409 & $\begin{array}{l}\text { Purine nucleoside } \\
\text { phosphorylase }\end{array}$ & PNPH_RAT & 376.3744403 & 32.28104 & 6.519143097 \\
\hline P1412 & Creatine kinase M-type & KCRM_RAT & 541.9422014 & 43.0178 & 6.632039261 \\
\hline P1413 & $\begin{array}{l}\text { L-lactate } \\
\text { dehydrogenase A chain }\end{array}$ & LDHA_RAT & 589.4044403 & 36.42734 & 9.279562822 \\
\hline P1529 & $\begin{array}{l}\text { Protein } \\
\text { disulfide-isomerase A3 }\end{array}$ & PDIA3_RAT & 699.4495433 & 57.04387 & 5.829417423 \\
\hline P1981 & $\begin{array}{l}\text { Guanine } \\
\text { nucleotide-binding } \\
\text { protein subunit } \\
\text { beta-2-like }\end{array}$ & GBLP_RAT & 395.3884018 & 35.51073 & 8.876677099 \\
\hline
\end{tabular}


Table 2. The primers were used for qPCR in this study.

\begin{tabular}{lll}
\hline Primer name & Type & Length Sequence $\left(5^{\prime}-3^{\prime}\right)$ \\
\hline Rat Pdia-3 & Forward & 20-mer GAGGCTTGCCCCTGAGTATG \\
\hline Rat Pdia-3 & Reverse & 19-mer GTTGGCAGTGCAATCCACC \\
\hline ERK1 & Forward & 20-mer AGCTGCTAAAGAGCCAGCAG \\
\hline ERK1 & Reverse & 20-mer GCAAGGCCAAAATCACAGAT \\
\hline Osteopontin & Forward & 20-mer GTTCTTGCACAGCTTCACCA \\
\hline Osteopontin & Reverse & 20-mer AAACAGCCCAGTGACCATTC \\
\hline $\begin{array}{lll}\text { Alkaline } \\
\text { phosphatase }\end{array}$ & Forward & 20-mer GACAAGAAGCCCTTCACAGC \\
\hline $\begin{array}{ll}\text { Alkaline } \\
\text { phosphatase }\end{array}$ & Reverse & 20-mer GGGGGATGTAGTTCTGCTCA \\
\hline MMP13 & Forward & 20-mer GAGGTGAAAAGGCTCAGTGC \\
\hline MMP13 & Reverse & 20-mer TGGGCCCATTGAAAAAGTAG \\
\hline
\end{tabular}

\section{2 weeks}

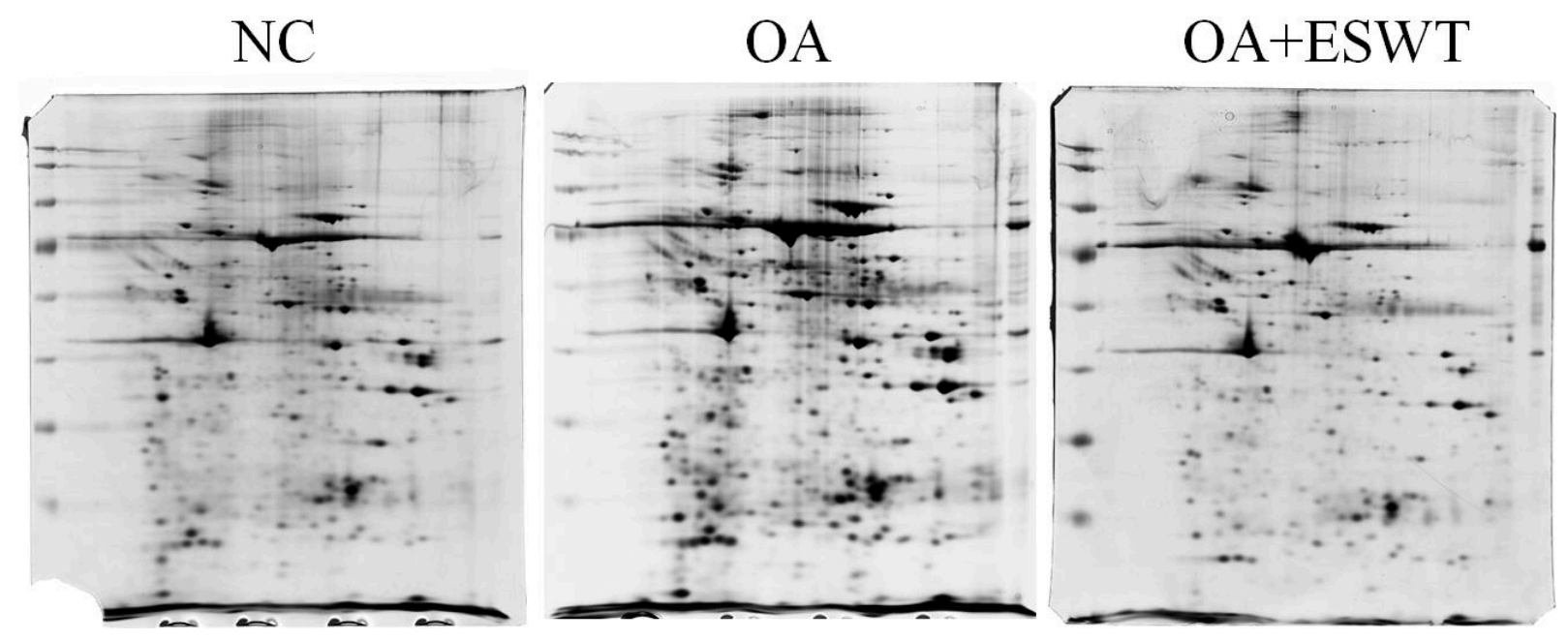

Supplemental Figure 1. The articular cartilage and subchondral bone of left knee were analysis by two-dimensional gel electrophoresis from NC, OA and OA+ESWT groups. 
A.

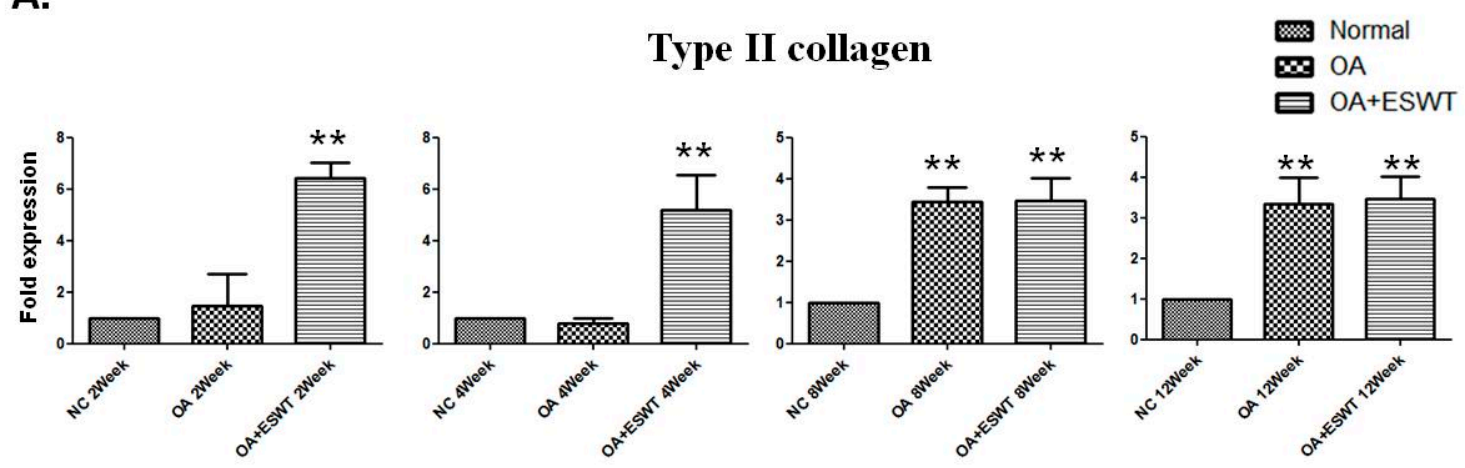

B.

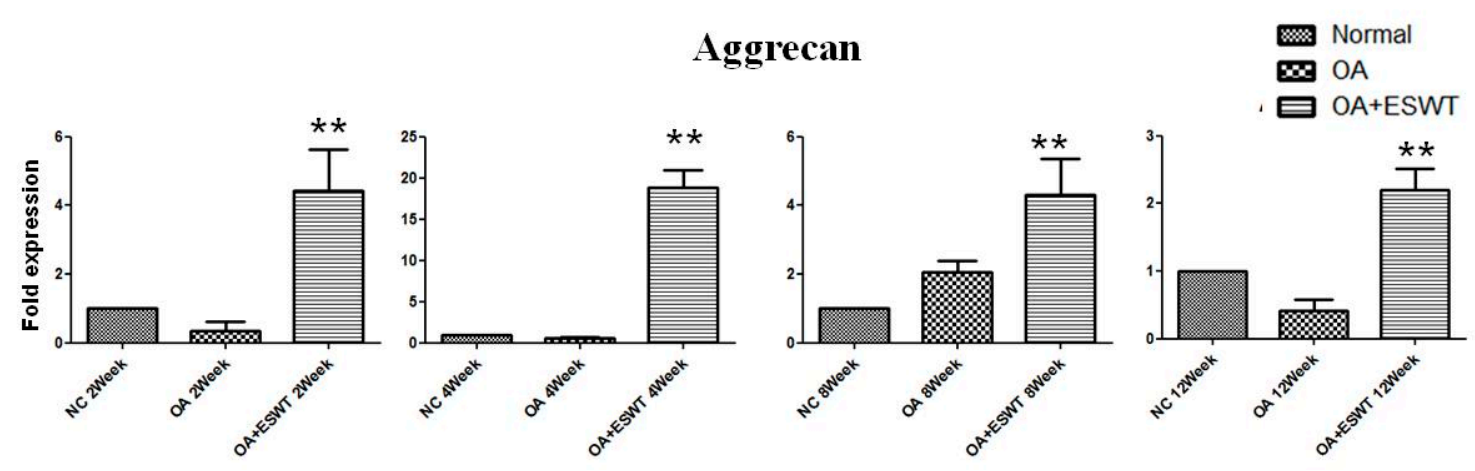

Supplemental Figure 2. The effect of shockwave therapy induced expression of cartilage related gene in early OA knee. The OA+ESWT group showed significant increases in expression of aggrecan and collagen type II when compared with $\mathrm{NC}$ and OA groups at 2, 48 and 12 weeks $(\mathrm{P}<0.05)$.

\section{Discussion}

In this study, we expanded the study and confirmed that osteoarthritic knee could be regressed by the application of shockwave therapy. Furthermore, we demonstrated a trend toward increase of Pdia-3 biosynthetic activity in response to pulsed acoustic energy released by shockwave therapy. The mitogenic and anabolic activities of osteoblast and chondrocyte increased relating to elevating ERK phosphorylation in sudchondral bone after ESWT. The growing evidence indicating that Pdia-3 dependent mechanisms are involved in rapid responses to the secosteroid 1, 25-dihydroxyvitamin $\mathrm{D} 3\left(1 \alpha, 25(\mathrm{OH})_{2} \mathrm{D}_{3}\right)$ signaling in osteoblast and chondrocyte cells $[12,19,20]$. Pdia-3 has been identified as a potential candidate as an alternate membrane-associated receptor for $1 \alpha, 25(\mathrm{OH})_{2} \mathrm{D}_{3}$. The $1 \alpha, 25(\mathrm{OH})_{2} \mathrm{D}_{3}$ directly regulates mineralization from the osteoblasts and matrix formation from the chondrocyte through classic vitamin D receptor (VDR) mediated genomic pathway and membrane receptor-mediated rapid responses via Pdia-3 dependent pathway. Our study provided the first evidence that the effect of ESWT on the OA knee revealed the regulation of protein Pdia-3, linking to osteoblast and chondrocyte cells integrity and function. This finding suggested the proposed biomechanical pathway was likely to be conserved of shockwave therapy in the treatment of OA knee.

It is well established that Pdia-3 mediated the membrane response to $1 \alpha, 25(\mathrm{OH})_{2} \mathrm{D}_{3}$, including phospholipase A2 (PLA2) stimulation dependent rapid release of prostaglandin E2 
(PGE2), activation of protein kinase $\mathrm{C}$ (PKC), then regulation of bone related gene transcription and mineralization via phosphorylation of transcription factors such as ERK1/2 in osteoblast-like MC3T3-E1 cells [19]. Jiaxuan et al found that in Pdia-3-silenced (Sh-Pdia-3) cells, $1 \alpha, 25(\mathrm{OH})_{2} \mathrm{D}_{3}$ failed to stimulate PKC and PGE2 reaction and in Pdia-3 overexpression cells (Ov-Pdia-3), the respond to $1 \alpha, 25(\mathrm{OH})_{2} \mathrm{D}_{3}$ were augmented. They suggested the Pdia3 could be a determining factor correlating directly with the magnitude of the membrane response to $1 \alpha, 25(\mathrm{OH})_{2} \mathrm{D}_{3}$ [32]. The principal findings of this study showed that application of ESWT induced Pdia-3 up-regulation leading to subchondral bone remolding after ACLT OA of knee in rats. The present gene expression showed the ability of the cells to mineralized their extracellular matrix and bone related genes, ALP, OPG and MMP13, significantly increased after shockwave therapy. The results were in agreement with prior studies had demonstrated that ESWT significantly enhances the osteogenic factors reflecting a local stimulation of bone formation during the fracture healing [33-35]. From the results presented, the data clearly showed that a number of genes encoding bone formation and relatively signaling molecules that could potentially transduce osteogenic effect in response to treatment with shockwave changing expression of Pdia3 in the subchondral bone of knee.

Pdia-3 mediated signaling results in gene transcription, which can also modulate bone formation. The key components of ERK1 in this signaling pathway were also activated. ERK had been found to act as an important mediator for mechanical-stimulated proliferation and differentiation of osteogenic cell. Previous studies revealed the ERK was involved in shockwave-augmented bone formation in segmental defects within 14 days after treatment. The phosphorylation of ERK is active throughout the period of ESW-induced bone regeneration and regulated the stimulation of biophysical shockwave therapy, triggering mitogenic and osteogenic responses in the defects [36]. Our present data reveals that the signals of ERK were active and it could play an important role in signaling subchondral bone remolding after 2 wk local application of shockwave therapy.

Several studies reported positive effects of ESWT in osteoarthritis of different joints in animals [37-41]. The exact mechanism of shockwave therapy was still unknown. Our current study provides the first evidence from immunohistochemistry that shockwave therapy can induce articular cartilages expression of Pdia-3, the critical transcription factor responsible for the matrix formation of chondrocyte. Recent studies reported that $1 \alpha, 25(\mathrm{OH})_{2} \mathrm{D}_{3}$ rapidly stimulated membrane signaling via Pdia-3 dependent activation in growth zone chondrocytes and promotes the production of matrix protein $[11,13,20,22,42,43]$. The present study showed the decrease of cartilage matrix loss and increased aggrecan and collagen II expression in shockwave group. It explained the biomolecular mechanism of shockwave therapy in cartilage development and maintenance of the chondrocyte phenotype. The regression of osteoarthritic knee was supported by the expression of Pdia-3 and biomarkers of the cartilage in the remolding surface of articular area.

The effect of shockwave therapy in the osteoarthritic rat knee showed time-dependent chondroprotection [44]. We observed that the most beneficial effects of shockwave therapy on the OA knee after 2 wk of shockwave application, and the effects of shockwave seemed to 
continue until 12 wks. The novel findings supported the concept that shockwave therapy provided a chondroprotective effect associated with improvement in subchondral bone remolding, significant decrease in the cartilage degradation and increase in chondrocyte activity in the initiation of ACLT OA. Application of shockwave therapy on the subchondral bone was effective in a time-dependent fashion in OA of the knee.

The exact mechanism of ESWT remains unexplored. The innovative findings in this study may unveil a new concept in the biomolecular pathway and treatment of osteoarthritis of the knee by ESWT. It appeared that local shockwave therapy application to the subchondral bone in medial tibia condyle affected the entire knee joint through stimulating the osteoblast and chondrocyte cells via up-regulation of Pdia-3.

There are some limitations in this study. The data obtained from this study were based on small animals' experiments. The results may differ in larger animals or human subjects. The dose conversion from small animals to larger animals or human subjects must be calculated with additional studies and clinical trial. The optimal ESWT dose and the ideal numbers of ESWT remain unknown. Furthermore, different manufacture companies used different indices of shockwave parameters, and the dose conversion formula among the different devices ae not readily available at the present time.

\section{Materials and Methods}

\subsection{Study Design}

The Institutional Review Board on animal experiment approved this study. All studies were performed in accordance with the guidelines in the study and the care of animals in experiment. All procedures and protocols were approved by the Institutional Animal Care and Use Committee of Chang Gung Memorial Hospital, Taiwan. One hundred forty-four male four-month-old Sprague-Dawley rats with body weight ranging from 250 to $275 \mathrm{mg}$ were used in this study. The rats were randomly divided into three groups with 48 rats in each group and each time course for 12 rats ( $n=48$ per group). The 12 rats were sacrificed at 2, 4, 8 and 12 weeks post-surgery. In 12 rats, six were for proteome analysis and six for histology study. The rats in normal control (NC) group received neither anterior cruciate ligament transection (ACLT) nor ESWT and served as the baseline control. The rats in OA group underwent ACLT, but received no ESWT. The rats in OA+ESWT group underwent ACLT and received ESWT ( 800 impulse at $\left.0.18 \mathrm{~mJ} / \mathrm{mm}^{2}\right)$ to the subchondral bone of the medial tibia condyle. 


\subsection{Experimental OA knee model}

Four-month-old male Sprague-Dawley rats were anesthetized using intraperitoneal injection of pentobarbital ( $50 \mathrm{mg} / \mathrm{Kg}$ body weight). The left knees of the animals underwent surgery comprising medial parapatellar arthrotomy and ACLT to induce ACLT-mediated knee OA as previously described [45]. Animals were allowed unrestricted weight-bearing and activity as tolerated. The left knee was excised after animals were sacrificed at each time course.

\subsection{Histology}

Whole knee joints were fixed in $4 \%$ phosphate buffered paraformaldehyde, decalcified in $10 \%$ phosphate buffered EDTA, then embedded in paraffin. Specimens were longitudinally cut into 4- $\mu \mathrm{m}$ sections for hematoxylin and eosin staining and Alcian blue staining (Sigma-Aldrich). Histomorphometry of articular cartilage was evaluated by a 14-point Mankin scoring system (for structural integrity, from $0=$ normal to $6=$ complete disorganization; for cells, from $0=$ normal to 3 = hypocellularity; for cartilage height, from $0=$ normal to $4=$ complete loss; for tidemark integrity, from $0=$ normal to $1=$ disruption) (25). Twelve sections from 6 rats were measured under 100x magnification using a Zeiss Axioskop 2 plus microscope (Carl Zeiss Microimaging) with a cool CCD camera and Image-Pro Plus image analysis software (SNAP-Pro c.f. Digital kit; Media Cybernetics). In some cases, the specimens were fixed in absolute alcohol, embedded in glycolmethylacrylate (Fluka), then cut longitudinally into 8 - $\mu \mathrm{m}$ sections using a rotary tungsten steel-bladed microtome for von Kossa's and tartrate-resistant acid phosphatase histochemical staining (Sigma-Aldrich). All sections were independently assessed by two individuals. Cartilage destruction was assessed using the Mankin scoring system [46]. Each-section was evaluated using Mankin's histological and histochemical grading system, including structural changes in all layers of the uncalcified cartilage, tidemark integrity and Safarine-O staining. Safarine-O staining is the indicator of proteoglycan amount in the extracellular matrix.

\subsection{Two-dimensional electrophoresis}

Samples of $250 \mu \mathrm{g}$ protein from 2 comparative subjects were applied on immobilized pH 3-10 nonlinear gradient strips with pretreated CyDye. Isoelectric focusing is performed by an Ettan ${ }^{\mathrm{TM}}$ IPGphor II/3. Proteins in the strips were separated in $15 \%$ SDS-PAGE and stained using fluorescence dyes. Protein spots in gels were scanned by an Amersham Image 
Scanner. Image, spot match and spot intensity were analyzed and calculated by a Bio-Rad Proteoweaver 2-D Analysis Software Version 4.0.

\subsection{MALDI-TOF mass spectrometry/LC mass spectrometry}

Spots of interest were excised and washed with $10 \mathrm{mM}$ ammonium bicarbonate and $50 \%$ acetonitrile in $10 \mathrm{mM}$ ammonium bicarbonate. After wash and shrinkage with acrtonitrile, the dried gels were digested by trypsin at $30^{\circ} \mathrm{C}$ for 4 hours. The trypsin digest was extracted by trifluoroacetate. Aliquots of the digest were loaded onto AnchorChip for one PMF from MALDI-TOF, and TOF/TOF MS/MS analysis of fragment peptides using the FlexControlTM software. Peptide mass data were submitted to NCBI and Swiss-Port database using MASCO search engines.

\subsection{Quantitative RT-PCR}

The primers of bone and cartilage biomarkers including osteoprotegerin (OPG), alkaline phosphatase (ALP), matrix metallopeptidase 13 (MMP13), collage type II and aggrecan were detected by quantitative RT-PCR (Table 2). Total RNA was extracted and purified from knee joint tissue using QIAzol reagent. Total RNA (1 $\mu \mathrm{g})$ was reverse transcribed onto cDNA. $25 \mu \mathrm{L}$ of PCR mixture containing cDNA template equivalent to 20 ng total RNA, $2.5 \mu \mathrm{M}$ each forward, reverse primer and 2X iQTM SYBR green supermix was amplified using the iCycler $\mathrm{iQ}{ }^{\circledR}$ Real time PCR detection system with an initial melt at $95^{\circ} \mathrm{C}$ for $5 \mathrm{~min}$ followed by 40 cycles at $94^{\circ} \mathrm{C}$ for $15 \mathrm{sec}, 52^{\circ} \mathrm{C}$ for $20 \mathrm{sec}$ and $72^{\circ} \mathrm{C}$ for 30 sec using following primer oligonucleotide sequences followed by PCR amplification using responsive molecules and rat $18 \mathrm{~S}$ rRNA primers (forward) (5'-GCAGCTAGGAATAATGGAATAGGA-3'),(reverse)(5'-TAATGAAAACATTCTTG GCAAATG-3'). The number of amplification steps required to each an arbitary intensity threshold $(\mathrm{Ct})$ is computed. The relative gene expression level was presented $2^{(-\Delta \mathrm{Ct})}$, where $\Delta \mathrm{Ct}=\mathrm{Ct}$ target-Ct $18 \mathrm{~S}$ rRNA. Fold change for the treatment was defined as the relative expression, compared with the vehicle and is calculated as $2^{-\Delta \Delta \mathrm{Ct}}$, where $\Delta \Delta \mathrm{Ct}=\Delta \mathrm{Ct}$ treatment $-\Delta \mathrm{Ct}$ vehicle.

\subsection{In situ immunohistochemistry}

Sections were hybridized with relative antibodies against candidate proteins and a nonbiotin HRP detection system (BioGenex). Immunoreactivity in specimens was demonstrated using a horseradish peroxidase (HRP)-3'-, 3'diaminobenzidine (DAB) cell and tissue staining kit (R\&D systems, Inc. Minneapolis, MN, USA) according to manufacturer's 
instructions. Antibodies against discovered molecules were used. Sections were than incubated with biotinylated secondary antibodies with streptavidin conjugated to HRP, followed by chromogen solution and counterstaining with hematoxylin. Sections were finally dehydrated and mounted. Sections without primary antibodies were enrolled as negative controls for the immunostaining. The number of positive immunolabeled cells and total cells in each area were counted and percentages of positive-labeled cells were presented. The osteoblasts and chondrocytes were identified morphologically. A pathologist, blinded to the treatment, performed the measurements of all sections under 100 fold magnifications.

\subsection{Statistical analysis}

All values were expressed as mean \pm standard error. One-way ANOVA and Tukey tests were used to assess the differences among the groups. The level of statistical significance was set at $\mathrm{P}<0.05$.

\section{Conclusions}

Our proteomic data revealed abundant significant Pdia-3 expression in joint tissue microenviorments may be actively responded to ESWT treatment, which potentially regulated biological function of chondrocytes and osteoblasts in OA knee. Furthermore, ESWT has the potential ability in the treatment of osteoarthritis of the knee.

Acknowledgments: This work was supported totally by a grant from Chang Gung Memorial hospital Research Grant (No: CMRPG8B1311 and CMRPG8B1312)

Author Contributions: Shan-Ling Hsu, Jai-Hong Cheng participated in the study with primary responsibility in conception and design drafting, overview the entire study, data collection and analysis, literature review, reference search, draft writing and critically revised the manuscript and read proof of the final manuscript.

Ching-Jen Wang, Jih-Yang Ko, and Chih-Hsiang Hsu participated in the study with primary duty in reference search, literature review and read proof of final manuscript.

Conflicts of Interest: The authors declared that they did not receive any honoraria or consultancy fees in writing this manuscript. No benefits in any form have been received or will be received from a commercial party related directly or indirectly to the subject of this article. One author (Chin-Jen Wang) serves as a member of the advisory committee of Sanuwave, (Alpharetta, GA) and this study is performed independent of the appointment. The remaining authors declared no conflict of interest. 


\section{References}

1. Theis, K. A.; Murphy, L.; Hootman, J. M.; Helmick, C. G.; Yelin, E., Prevalence and correlates of arthritis-attributable work limitation in the US population among persons ages 18-64: 2002 National Health Interview Survey Data. Arthritis and rheumatism 2007, 57, (3), 355-63.

2. Kurz, B.; Lemke, A. K.; Fay, J.; Pufe, T.; Grodzinsky, A. J.; Schunke, M., Pathomechanisms of cartilage destruction by mechanical injury. Annals of anatomy = Anatomischer Anzeiger : official organ of the Anatomische Gesellschaft 2005, 187, (5-6), 473-85.

3. Min, J. L.; Meulenbelt, I.; Kloppenburg, M.; van Duijn, C. M.; Slagboom, P. E., Mutation analysis of candidate genes within the 2q33.3 linkage area for familial early-onset generalised osteoarthritis. European journal of human genetics : EJHG 2007, 15, (7), 791-9.

4. Haywood, L.; McWilliams, D. F.; Pearson, C. I.; Gill, S. E.; Ganesan, A.; Wilson, D.; Walsh, D. A., Inflammation and angiogenesis in osteoarthritis. Arthritis and rheumatism 2003, 48, (8), 2173-7.

5. Bondeson, J.; Wainwright, S. D.; Lauder, S.; Amos, N.; Hughes, C. E., The role of synovial macrophages and macrophage-produced cytokines in driving aggrecanases, matrix metalloproteinases, and other destructive and inflammatory responses in osteoarthritis. Arthritis research \& therapy 2006, 8, (6), R187.

6. Blom, A. B.; van Lent, P. L.; Libregts, S.; Holthuysen, A. E.; van der Kraan, P. M.; van Rooijen, N.; van den Berg, W. B., Crucial role of macrophages in matrix metalloproteinase-mediated cartilage destruction during experimental osteoarthritis: involvement of matrix metalloproteinase 3. Arthritis and rheumatism 2007, 56, (1), 147-57.

7. Yudoh, K.; Nguyen v, T.; Nakamura, H.; Hongo-Masuko, K.; Kato, T.; Nishioka, K., Potential involvement of oxidative stress in cartilage senescence and development of osteoarthritis: oxidative stress induces chondrocyte telomere instability and downregulation of chondrocyte function. Arthritis research \& therapy 2005, 7, (2), R380-91.

8. Kim, H. A.; Lee, Y. J.; Seong, S. C.; Choe, K. W.; Song, Y. W., Apoptotic chondrocyte death in human osteoarthritis. The Journal of rheumatology 2000, 27, (2), 455-62.

9. Hashimoto, S.; Setareh, M.; Ochs, R. L.; Lotz, M., Fas/Fas ligand expression and induction of apoptosis in chondrocytes. Arthritis and rheumatism 1997, 40, (10), 1749-55.

10. Johnson, K.; Svensson, C. I.; Etten, D. V.; Ghosh, S. S.; Murphy, A. N.; Powell, H. C.; Terkeltaub, R., Mediation of spontaneous knee osteoarthritis by progressive chondrocyte ATP depletion in Hartley guinea pigs. Arthritis and rheumatism 2004, 50, (4), 1216-25.

11. Sylvia, V. L.; Del Toro, F.; Dean, D. D.; Hardin, R. R.; Schwartz, Z.; Boyan, B. D., Effects of 1alpha,25-(OH)(2)D(3) on rat growth zone chondrocytes are mediated via 
cyclooxygenase-1 and phospholipase A(2). Journal of cellular biochemistry. Supplement 2001, Suppl 36, 32-45.

12. Boyan, B. D.; Chen, J.; Schwartz, Z., Mechanism of Pdia3-dependent 1alpha,25-dihydroxy vitamin D3 signaling in musculoskeletal cells. Steroids 2012, 77, (10), 892-6.

13. Doroudi, M.; Boyan, B. D.; Schwartz, Z., Rapid 1alpha,25(OH)(2)D (3) membrane-mediated activation of $\mathrm{Ca}(2)(+) /$ calmodulin-dependent protein kinase II in growth plate chondrocytes requires Pdia3, PLAA and caveolae. Connective tissue research 2014, 55 Suppl 1, 125-8.

14. Wang, C. J.; Ko, J. Y.; Kuo, Y. R.; Yang, Y. J., Molecular changes in diabetic foot ulcers. Diabetes research and clinical practice 2011, 94, (1), 105-10.

15. Wang, C. J.; Weng, L. H.; Ko, J. Y.; Sun, Y. C.; Yang, Y. J.; Wang, F. S., Extracorporeal shockwave therapy shows chondroprotective effects in osteoarthritic rat knee. Archives of orthopaedic and trauma surgery 2011, 131, (8), 1153-8.

16. Liu, W.; He, J.; Lin, R.; Liang, J.; Luo, Q., Differential proteomics of the synovial membrane between bilateral and unilateral knee osteoarthritis in surgeryinduced rabbit models. Molecular medicine reports 2016.

17. Liao, W.; Li, Z.; Zhang, H.; Li, J.; Wang, K.; Yang, Y., Proteomic analysis of synovial fluid as an analytical tool to detect candidate biomarkers for knee osteoarthritis. International journal of clinical and experimental pathology 2015, 8, (9), 9975-89.

18. Ruiz-Romero, C.; Blanco, F. J., The role of proteomics in osteoarthritis pathogenesis research. Current drug targets 2009, 10, (6), 543-56.

19. Chen, J.; Doroudi, M.; Cheung, J.; Grozier, A. L.; Schwartz, Z.; Boyan, B. D., Plasma membrane Pdia3 and VDR interact to elicit rapid responses to 1alpha,25(OH)(2)D(3). Cellular signalling 2013, 25, (12), 2362-73.

20. Doroudi, M.; Olivares-Navarrete, R.; Boyan, B. D.; Schwartz, Z., A review of 1alpha,25(OH)2D3 dependent Pdia3 receptor complex components in Wnt5a non-canonical pathway signaling. The Journal of steroid biochemistry and molecular biology 2015, 152, 84-8.

21. Doroudi, M.; Olivares-Navarrete, R.; Hyzy, S. L.; Boyan, B. D.; Schwartz, Z., Signaling components of the 1alpha,25(OH)2D3-dependent Pdia3 receptor complex are required for Wnt5a calcium-dependent signaling. Biochimica et biophysica acta 2014, 1843, (11), 2365-75.

22. Doroudi, M.; Schwartz, Z.; Boyan, B. D., Phospholipase A2 activating protein is required for 1alpha,25-dihydroxyvitamin D3 dependent rapid activation of protein kinase $\mathrm{C}$ via Pdia3. The Journal of steroid biochemistry and molecular biology 2012, 132, (1-2), 48-56.

23. Avbersek-Luznik, I.; Gmeiner Stopar, T.; Marc, J., Activity or mass concentration of bone-specific alkaline phosphatase as a marker of bone formation. Clinical chemistry and laboratory medicine 2007, 45, (8), 1014-8. 
24. van Straalen, J. P.; Sanders, E.; Prummel, M. F.; Sanders, G. T., Bone-alkaline phosphatase as indicator of bone formation. Clinica chimica acta; international journal of clinical chemistry 1991, 201, (1-2), 27-33.

25. Tanaka, H.; Mine, T.; Ogasa, H.; Taguchi, T.; Liang, C. T., Expression of RANKL/OPG during bone remodeling in vivo. Biochemical and biophysical research communications 2011, 411, (4), 690-4.

26. Simonet, W. S.; Lacey, D. L.; Dunstan, C. R.; Kelley, M.; Chang, M. S.; Luthy, R.; Nguyen, H. Q.; Wooden, S.; Bennett, L.; Boone, T.; Shimamoto, G.; DeRose, M.; Elliott, R.; Colombero, A.; Tan, H. L.; Trail, G.; Sullivan, J.; Davy, E.; Bucay, N.; Renshaw-Gegg, L.; Hughes, T. M.; Hill, D.; Pattison, W.; Campbell, P.; Sander, S.; Van, G.; Tarpley, J.; Derby, P.; Lee, R.; Boyle, W. J., Osteoprotegerin: a novel secreted protein involved in the regulation of bone density. Cell 1997, 89, (2), 309-19.

27. Udagawa, N.; Takahashi, N.; Yasuda, H.; Mizuno, A.; Itoh, K.; Ueno, Y.; Shinki, T.; Gillespie, M. T.; Martin, T. J.; Higashio, K.; Suda, T., Osteoprotegerin produced by osteoblasts is an important regulator in osteoclast development and function. Endocrinology 2000, 141, (9), 3478-84.

28. Tuckermann, J. P.; Pittois, K.; Partridge, N. C.; Merregaert, J.; Angel, P., Collagenase-3 (MMP-13) and integral membrane protein 2a (Itm2a) are marker genes of chondrogenic/osteoblastic cells in bone formation: sequential temporal, and spatial expression of Itm2a, alkaline phosphatase, MMP-13, and osteocalcin in the mouse. Journal of bone and mineral research : the official journal of the American Society for Bone and Mineral Research 2000, 15, (7), 1257-65.

29. Hayami, T.; Kapila, Y. L.; Kapila, S., MMP-1 (collagenase-1) and MMP-13 (collagenase-3) differentially regulate markers of osteoblastic differentiation in osteogenic cells. Matrix biology : journal of the International Society for Matrix Biology 2008, 27, (8), 682-92.

30. Dufield, D. R.; Nemirovskiy, O. V.; Jennings, M. G.; Tortorella, M. D.; Malfait, A. M.; Mathews, W. R., An immunoaffinity liquid chromatography-tandem mass spectrometry assay for detection of endogenous aggrecan fragments in biological fluids: Use as a biomarker for aggrecanase activity and cartilage degradation. Analytical biochemistry 2010, 406, (2), 113-23.

31. Fraser, A.; Fearon, U.; Billinghurst, R. C.; Ionescu, M.; Reece, R.; Barwick, T.; Emery, P.; Poole, A. R.; Veale, D. J., Turnover of type II collagen and aggrecan in cartilage matrix at the onset of inflammatory arthritis in humans: relationship to mediators of systemic and local inflammation. Arthritis and rheumatism 2003, 48, (11), 3085-95.

32. Chen, J.; Lobachev, K. S.; Grindel, B. J.; Farach-Carson, M. C.; Hyzy, S. L.; El-Baradie, K. B.; Olivares-Navarrete, R.; Doroudi, M.; Boyan, B. D.; Schwartz, Z., Chaperone properties of pdia3 participate in rapid membrane actions of 1alpha,25-dihydroxyvitamin d3. Molecular endocrinology 2013, 27, (7), 1065-77.

33. Kaspar, D.; Neidlinger-Wilke, C.; Holbein, O.; Claes, L.; Ignatius, A., Mitogens are increased in the systemic circulation during bone callus healing. Journal of 
orthopaedic research : official publication of the Orthopaedic Research Society 2003, 21, (2), 320-5.

34. Taniguchi, T.; Matsumoto, T.; Shindo, H., Changes of serum levels of osteocalcin, alkaline phosphatase, IGF-I and IGF-binding protein-3 during fracture healing. Injury 2003, 34, (7), 477-9.

35. Wang, F. S.; Wang, C. J.; Sheen-Chen, S. M.; Kuo, Y. R.; Chen, R. F.; Yang, K. D., Superoxide mediates shock wave induction of ERK-dependent osteogenic transcription factor (CBFA1) and mesenchymal cell differentiation toward osteoprogenitors. The Journal of biological chemistry 2002, 277, (13), 10931-7.

36. Chen, Y. J.; Kuo, Y. R.; Yang, K. D.; Wang, C. J.; Sheen Chen, S. M.; Huang, H. C.; Yang, Y. J.; Yi-Chih, S.; Wang, F. S., Activation of extracellular signal-regulated kinase (ERK) and p38 kinase in shock wave-promoted bone formation of segmental defect in rats. Bone 2004, 34, (3), 466-77.

37. Dahlberg, J.; Fitch, G.; Evans, R. B.; McClure, S. R.; Conzemius, M., The evaluation of extracorporeal shockwave therapy in naturally occurring osteoarthritis of the stifle joint in dogs. Veterinary and comparative orthopaedics and traumatology : V.C.O.T 2005, 18, (3), 147-52.

38. Frisbie, D. D.; Kawcak, C. E.; McIlwraith, C. W., Evaluation of the effect of extracorporeal shock wave treatment on experimentally induced osteoarthritis in middle carpal joints of horses. American journal of veterinary research 2009, 70, (4), 449-54.

39. Mueller, M.; Bockstahler, B.; Skalicky, M.; Mlacnik, E.; Lorinson, D., Effects of radial shockwave therapy on the limb function of dogs with hip osteoarthritis. The Veterinary record 2007, 160, (22), 762-5.

40. Ochiai, N.; Ohtori, S.; Sasho, T.; Nakagawa, K.; Takahashi, K.; Takahashi, N.; Murata, R.; Takahashi, K.; Moriya, H.; Wada, Y.; Saisu, T., Extracorporeal shock wave therapy improves motor dysfunction and pain originating from knee osteoarthritis in rats. Osteoarthritis and cartilage / OARS, Osteoarthritis Research Society 2007, 15, (9), 1093-6.

41. Revenaugh, M. S., Extracorporeal shock wave therapy for treatment of osteoarthritis in the horse: clinical applications. The Veterinary clinics of North America. Equine practice 2005, 21, (3), 609-25, vi.

42. Schwartz, Z.; Graham, E. J.; Wang, L.; Lossdorfer, S.; Gay, I.; Johnson-Pais, T. L.; Carnes, D. L.; Sylvia, V. L.; Boyan, B. D., Phospholipase A2 activating protein (PLAA) is required for 1alpha,25(OH)2D3 signaling in growth plate chondrocytes. Journal of cellular physiology 2005, 203, (1), 54-70.

43. Boyan, B. D.; Wong, K. L.; Wang, L.; Yao, H.; Guldberg, R. E.; Drab, M.; Jo, H.; Schwartz, Z., Regulation of growth plate chondrocytes by 1,25-dihydroxyvitamin D3 requires caveolae and caveolin-1. Journal of bone and mineral research : the official journal of the American Society for Bone and Mineral Research 2006, 21, (10), 1637-47.

44. Wang, C. J.; Sun, Y. C.; Wong, T.; Hsu, S. L.; Chou, W. Y.; Chang, H. W., Extracorporeal shockwave therapy shows time-dependent chondroprotective effects 
in osteoarthritis of the knee in rats. The Journal of surgical research 2012, 178, (1), 196-205.

45. Hayami, T.; Pickarski, M.; Zhuo, Y.; Wesolowski, G. A.; Rodan, G. A.; Duong, L. T., Characterization of articular cartilage and subchondral bone changes in the rat anterior cruciate ligament transection and meniscectomized models of osteoarthritis. Bone 2006, 38, (2), 234-43.

46. Mankin, H. J.; Dorfman, H.; Lippiello, L.; Zarins, A., Biochemical and metabolic abnormalities in articular cartilage from osteo-arthritic human hips. II. Correlation of morphology with biochemical and metabolic data. The Journal of bone and joint surgery. American volume 1971, 53, (3), 523-37.

C 2016 by the authors; licensee Preprints, Basel, Switzerland. This article is an open access article distributed under the terms and conditions of the Creative Commons by Attribution (CC-BY) license (http://creativecommons.org/licenses/by/4.0/). 